\title{
AC-Coupled Front-End for Biopotential Measurements
}

\author{
Enrique Mario Spinelli*, Student Member, IEEE, Ramon Pallàs-Areny, Fellow, IEEE, and \\ Miguel Angel Mayosky, Senior Member, IEEE
}

\begin{abstract}
AC coupling is essential in biopotential measurements. Electrode offset potentials can be several orders of magnitude larger than the amplitudes of the biological signals of interest, thus limiting the admissible gain of a dc-coupled front end to prevent amplifier saturation. A high-gain input stage needs ac input coupling. This can be achieved by series capacitors, but in order to provide a bias path, grounded resistors are usually included, which degrade the common mode rejection ratio (CMRR). This paper proposes a novel balanced input ac-coupling network that provides a bias path without any connection to ground, thus resulting in a high CMRR. The circuit being passive, it does not limit the differential dc input voltage. Furthermore, differential signals are ac coupled, whereas common-mode voltages are dc coupled, thus allowing the closed-loop control of the dc common mode voltage by means of a driven-right-leg circuit. This makes the circuit compatible with common-mode de shifting strategies intended for single-supply biopotential amplifiers. The proposed circuit allows the implementation of high-gain biopotential amplifiers with a reduced number of parts, thus resulting in low power consumption. An electrocardiogram amplifier built according to the proposed design achieves a CMRR of $123 \mathrm{~dB}$ at $50 \mathrm{~Hz}$.
\end{abstract}

Index Terms-AC coupling, biopotential amplifiers, electrode offset potential.

\section{INTRODUCTION}

A COMMON front-end in biopotential measurements is a dc-coupled fully differential amplifier followed by a difference amplifier, as in the classical three op-amp instrumentation amplifier. If the input stage has a large gain, this circuit achieves a high common mode rejection ratio (CMRR) without any trimmings [1]. Furthermore, the equivalent input noise only depends on the two op-amps constituting the fully differential amplifier. These are coveted features in biopotential amplifiers, but due to electrode offset voltages, the overall gain is limited to moderate values [2]. This situation is even worse in low-voltage applications such as battery-powered amplifiers. Moreover, as the first-stage gain is reduced, subsequent stages are needed to

Manuscript received February 7, 2002; revised November 8, 2002. Asterisk indicates corresponding author.

${ }^{*}$ E. M. Spinelli is with the Laboratorio de Electrónica Industrial, Control e Instrumentación (LEICI), Departamento de Electrotecnia, Universidad Nacional de La Plata (UNLP), and also with the Comisión de Investigaciones Científicas de la Provincia de Buenos Aires (CICPBA) CC 91, 1900 La Plata, Argentina (spinelli@ing.unlp.edu.ar)

R. Pallàs-Areny is with the Departament d'Enginyeria Electrònica, Universitat Politècnica de Catalunya (UPC) EPS Castelldefels, 08860 Castelldefels (Barcelona), Spain.

M. A. Mayosky is with the Laboratorio de Electrónica Industrial, Control e Instrumentación (LEICI), Departamento de Electrotecnia, Universidad Nacional de La Plata (UNLP), and also with the Comisión de Investigaciones Científicas de la Provincia de Buenos Aires (CICPBA), CC 91, 1900 La Plata, Argentina.

Digital Object Identifier 10.1109/TBME.2003.808826

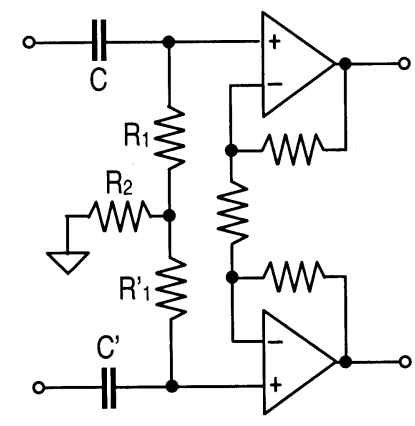

(a)

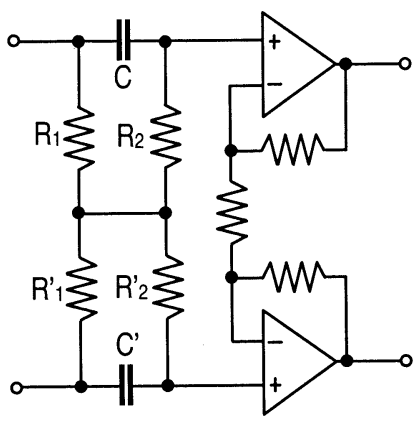

(b)
Fig. 1. (a) Typical circuit for balanced ac coupling. (b) Proposed ac-coupling circuit without any grounding resistor.

obtain a high gain, thus increasing the number of components and, hence, power consumption.

A high-gain front-end amplifier for biopotentials needs input ac coupling. The simplest ac-coupling technique is a passive high-pass filter in front of a dc amplifier. Because biopotential amplifiers are usually differential, that filter must be differential as well. Fig. 1(a) shows a typical differential filter for ac coupling [3]. This circuit is simple and suits low-power applications, but resistor $R_{2}$ reduces the input common mode impedance $Z_{\mathrm{C}}$, which degrades the effective CMRR due to the potential divider effect [4]. The CMRR of this coupling network depends on component tolerance, it is strongly degraded by electrode's impedance unbalances and decreases for increasing frequency.

For the circuit of Fig. 1(a), both CMRR and $Z_{C}$ increase with $R_{2}$. However, $R_{2}$ cannot be spared (infinite value), because the amplifier needs a bias path. The effective value of grounded resistors can be increased by bootstraping [5], but this solution amplifies op amps' offset more than the signal, hence, limiting the admissible gain of the front end.

This paper presents a simple ac-coupled front end that does not require any grounded resistor, thus achieving a large CMRR.

\section{Proposed Circuit}

The proposed front end includes the novel, balanced accoupling network shown in Fig. 1(b). This circuit provides ac coupling for differential signals and a dc path for amplifier bias currents, which drain to ground through the third (common) biopotential electrode. Hence, this solution cannot be applied to two-electrode amplifiers. Because the input network is not grounded, if a common mode input voltage is applied, no currents flow through the network (there is not any path for 


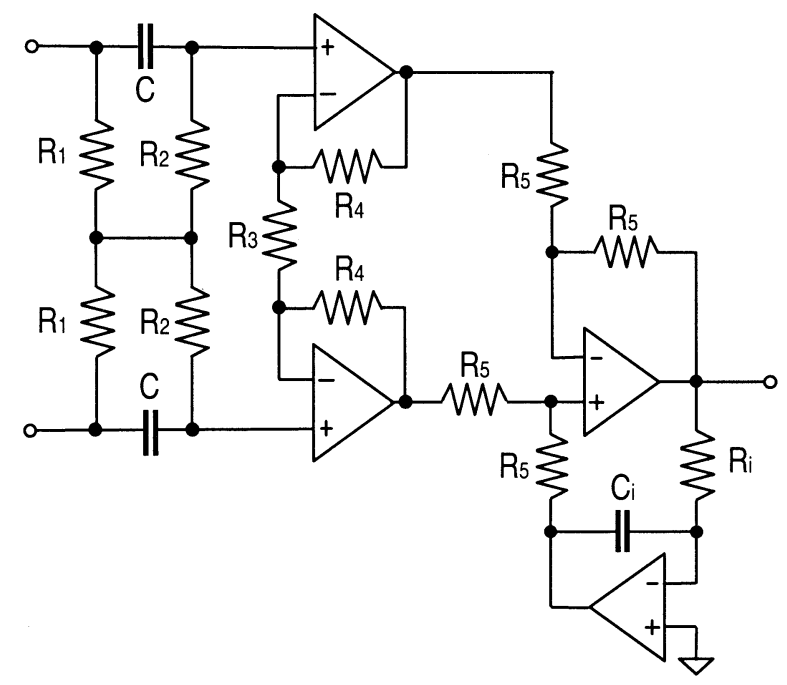

Fig. 2. Proposed amplifier circuit, which has two ac-coupling stages, a passive input stage, and an active dc suppression circuit.

common mode currents), so that all network's nodes achieve the same potential. This absence of potential difference due to common mode inputs implies an ideally infinite CMRR regardless of component tolerances. In practice, however, there are some grounded impedances not included in the model (i.e. op amps' input capacitances), and because of unbalanced electrode impedances, the CMRR becomes finite. Nevertheless, this limitation is not attributable to the ac-coupling network.

If $R_{2} C=R_{2}^{\prime} C^{\prime}=\tau_{2}$ (see the Appendix), the transfer function of the ac-coupling network is

$$
G_{\mathrm{DD}}(s)=\frac{s \tau_{2}}{1+s \tau_{2}}
$$

that corresponds to a first-order high-pass filter. If the time constants are not matched, the circuit exhibits two poles and two zeros. Nevertheless, using passive components with a reasonable tolerance yields a transfer function quite close to (1) (see the Appendix). Anyway, that mismatch does not degrade the CMRR.

Because the transfer function (1) does not depend on $R_{1}$, one design criterion is to select it as high as practical in order to avoid loading effects on the input signal. Alternatively, selecting $R_{1}=R_{2}=R$ simplifies the design. According to [7, Sec. 4.2.3.2], we need an input impedance larger than $2.44 \mathrm{M} \Omega$ at $10 \mathrm{~Hz} . R=5 \mathrm{M} \Omega$ largely fulfills this requirement.

The circuit in Fig. 1(b) removes dc input voltages, but the op amps input offset voltages are amplified as input signals and can significantly reduce the output dynamic range. For example, a $1-\mathrm{mV}$ difference in input offset voltage and a gain of 1000 yield $1 \mathrm{~V}$ at the amplifier output. Moreover, the thermal noise of $R_{2}$ and $R_{2}^{\prime}$ from dc to the corner frequency $f_{\mathrm{c}}\left(=1 / 2 \pi R_{2} C\right)$, and op amps input voltage noise, including $1 / f$ noise, will be amplified. To remove offset voltage and reduce $1 / f$ noise, the amplifier itself must reject low frequencies. Fig. 2 shows a solution that uses an integrator in a feedback loop around the difference amplifier [3], [6].
The system has two ac-coupled stages: the front differential ac-coupling network and the high-pass difference amplifier. The overall transfer function is

$$
T(s)=\frac{s \tau_{2}}{\left(1+s \tau_{2}\right)} \frac{s \tau_{i} A_{V 0}}{\left(1+s \tau_{i}\right)}
$$

where $\tau_{i}=R_{i} C_{i}$ and $A_{V 0}=1+2 R_{4} / R_{3}$. The first factor in (2) corresponds to the passive ac-coupling network and the second factor corresponds to the amplifier and dc restoration circuits.

\section{A. Design Example: Electrocardiogram (ECG) Amplifier}

1) Low-Frequency Response: The low-frequency behavior of the proposed amplifier is defined by $\tau_{2}$ and $\tau_{i}$. These time constants are designed to obtain the desired transient response, which is usually given in terms of responses to rectangular or triangular pulses. For example, a $60 \mu \mathrm{V} \cdot \mathrm{s}$ impulse (e.g., a rectangular pulse of $1-\mathrm{mV}$ amplitude and 60-ms duration) shall not produce an offset on the electrocardiogram (ECG) record from the isoelectric line greater than $20 \mu \mathrm{V}$. The proposed circuit includes two cascaded ac stages both of which contribute to that offset, whose amplitude will be

$$
\begin{aligned}
& y(60 \mathrm{~ms})=\frac{1 \mathrm{mV}}{\tau_{2}-\tau_{i}}\left[\left(\tau_{i}-\tau_{2}\right)\right. \\
& \left.\quad+\tau_{2} e^{\frac{-60 \mathrm{~ms}}{\tau_{i}}}-\tau_{i} e^{\frac{-60 \mathrm{~ms}}{\tau_{2}}}\right] .
\end{aligned}
$$

A possible choice to achieve $y(60 \mathrm{~ms})<20 \mu \mathrm{V}$ is $\tau_{2}=4.7 \mathrm{~s}$ and $\tau_{i}=10 \mathrm{~s}$, that yields $y(60 \mathrm{~ms})=18.6 \mu \mathrm{V}$.

2) High-Frequency Response: The ECG amplifier must include a low-pass filter for bandwidth limiting. A second-order filter with a double pole (critical damping, no overshoot in the step response) is a good choice because it will not change the previously calculated overshoot. Otherwise, its transient response could increment $y(60 \mathrm{~ms})$, thus leading to an iterative design process. A double pole at $150 \mathrm{~Hz}$, yields attenuations of $3 \mathrm{~dB}$ at $100 \mathrm{~Hz}$ and $6 \mathrm{~dB}$ at $150 \mathrm{~Hz}$, which fulfill the requirements in [7]. The cutoff frequency was selected at $156 \mathrm{~Hz}$. The complete transfer function is then

$$
T(s)=\frac{s \tau_{2}}{\left(1+s \tau_{2}\right)} \frac{s \tau_{i} A_{V 0}}{\left(1+s \tau_{i}\right)} \frac{1}{\left(1+s \tau_{F}\right)^{2}}
$$

which, with the designed time-constant values, becomes

$$
T(s)=\frac{s 4.7}{(1+s 4.7)} \frac{s A_{V 0} 10}{(1+s 10)} \frac{1}{(1+s 0.001)^{2}} .
$$

The nominal amplifier gain was 1001 , obtained with $R_{4}=$ $200 \mathrm{k} \Omega$ and $R_{3}=400 \Omega$.

\section{B. Operational-Amplifier Considerations}

Because the amplifier concentrates its gain in the first stage, its equivalent input noise is determined by the op amps composing the input stage. Low-noise applications [i.e. high-resolution ECG, electroencephalogram (EEG)] require low noise input op amps. In battery-powered devices, low-power rail-to-rail op amps are an attractive choice. The proposed design has been implemented using the low-noise rail-to-rail TLC2274. 


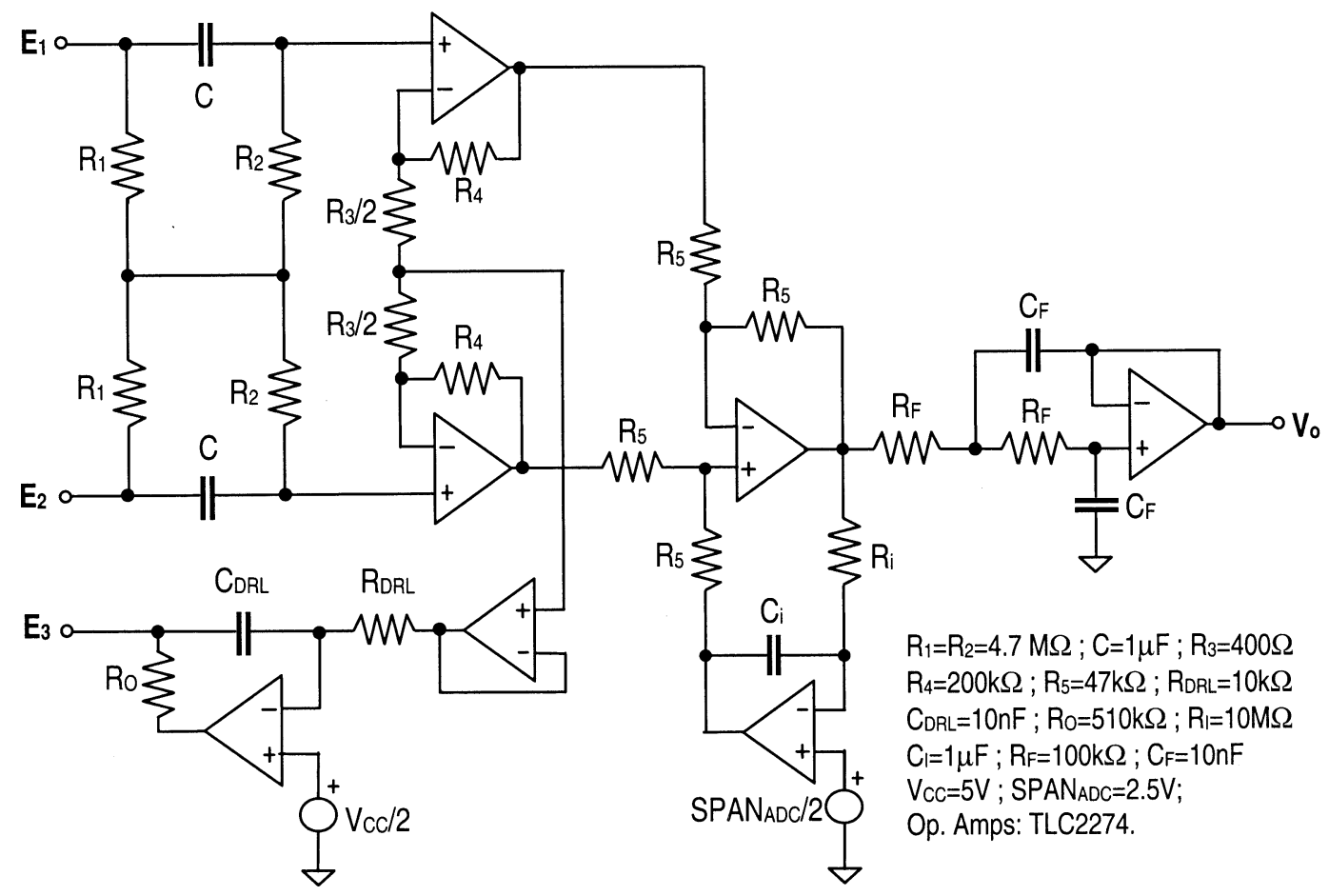

Fig. 3. Single-supply ECG amplifier based on the proposed circuit.

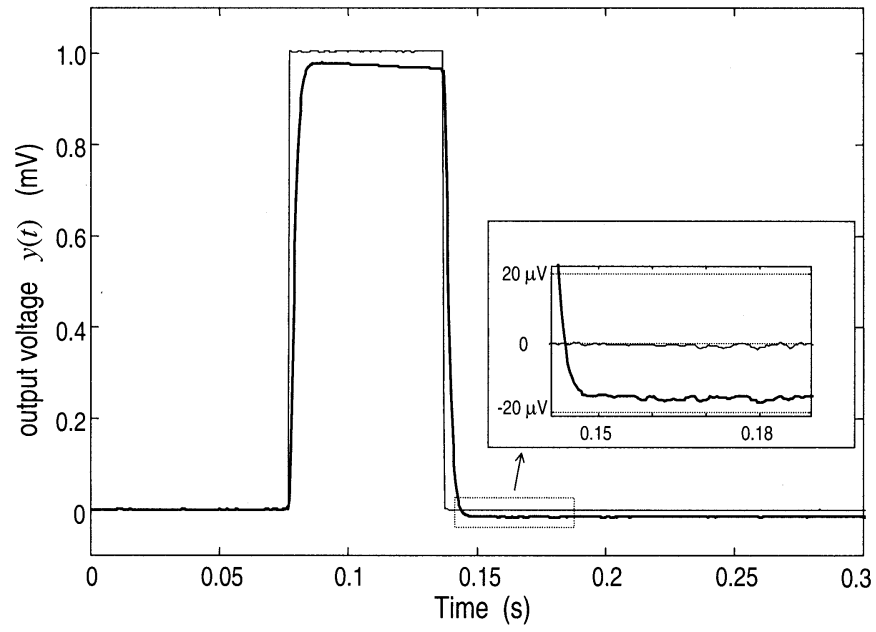

Fig. 4. Response of the ECG amplifier in Fig. 3 to a 1-mV, 60-ms rectangular pulse. The insert shows that the offset from the isoelectric line is less than $20 \mu \mathrm{V}$.

\section{EXPERIMENTAL RESULTS}

Fig. 3 shows the complete circuit implemented, which includes a driven-right-leg (DRL) circuit. The reference voltage connected to the noninverting input of the op amps of the DRL and the dc restoration circuits are positive to enable single-supply operation [8]. This strategy for single-supply operation is possible because the proposed coupling network in Fig. 1(b) provides ac coupling for differential signals but dc coupling for common-mode signals.

Fig. 4 shows the pulse response of the amplifier and Fig. 5 shows the frequency response. Both experimental results agree with the predictions by (3) and (5). There is no overshoot and the undershoot is less than $20 \mu \mathrm{V}$, as required. The relative am-

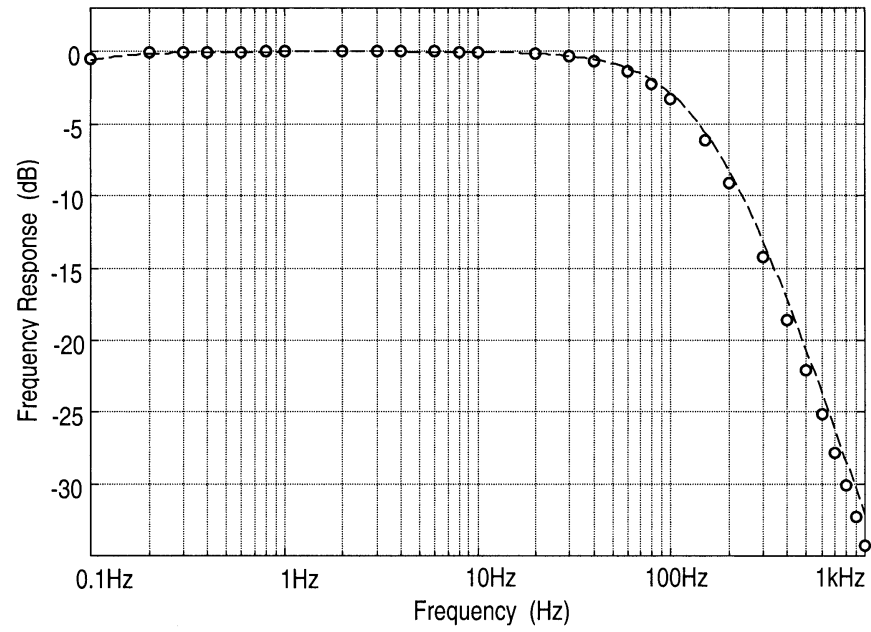

Fig. 5. Frequency response of the ECG amplifier in Fig. 3. The analytic expression is in dashed line and experimental data with markers.

plitude response for sinusoidal inputs falls within the tolerance bands defined in [7, Sec. 4.2.7.3].

Fig. 6 shows the test circuit for the CMRR, which includes impedance imbalance and cable capacitance $(\approx 200 \mathrm{pF})$. The overall measurement circuit was shielded in order to avoid power line interference external to the test and the measured CMRR at $50 \mathrm{~Hz}$ was $123 \mathrm{~dB}$, which exceeds the $60-\mathrm{dB}$ requirement in [7, Sec. 4.2.3.4].

\section{CONCLUSION}

The simple, novel, ac-coupled front end for biopotential measurements in Fig. 2 is a fully differential passive-coupling network that does not include any grounded resistor, hence, re- 


$$
G_{\mathrm{DD}}(s)=\frac{s\left\{\left(R_{1} \tau_{2}+R_{1}^{\prime} \tau_{2}^{\prime}\right)+s\left[R_{1} \tau_{2}\left(\tau_{1}^{\prime}+\tau_{2}^{\prime}\right)+R_{1}^{\prime} \tau_{2}^{\prime}\left(\tau_{1}+\tau_{2}\right)\right]\right\}}{s^{2}\left[R_{1} \tau_{2}\left(\tau_{1}^{\prime}+\tau_{2}^{\prime}\right)+R_{1}^{\prime} \tau_{2}^{\prime}\left(\tau_{1}+\tau_{2}\right)\right]+s\left[R_{1} \tau_{2}+R_{1}\left(\tau_{1}^{\prime}+\tau_{2}^{\prime}\right)+R_{1}^{\prime}\left(\tau_{1}+\tau_{2}\right)+R_{1}^{\prime} \tau_{2}^{\prime}\right]+\left[R_{1}+R_{1}^{\prime}\right]}
$$

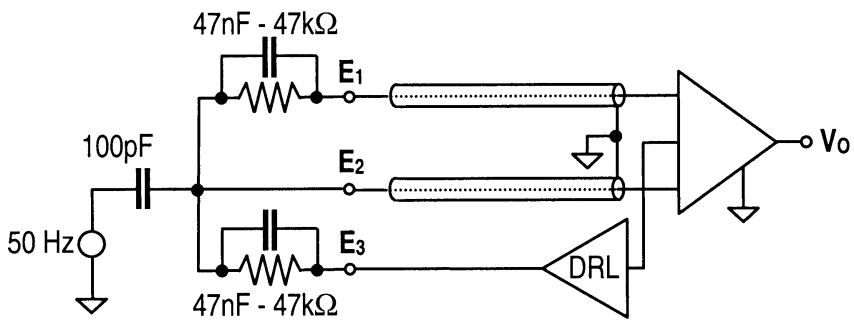

Fig. 6. Circuit to test the CMMR.

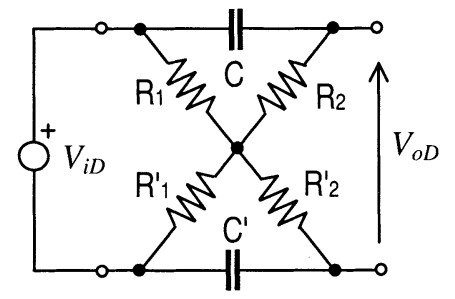

Fig. 7. Proposed fully differential ac-coupling network that does not include any grounded resistor.

sulting in a high CMRR. The proposed network enables the design of a high gain for the input stage of biopotential measurement systems, thus leading to implementations with a reduced number of stages, which are particularly convenient for lowpower applications. Because the common-mode voltage is dc coupled, the proposed circuit also suits single-supply operation.

A single-supply ECG amplifier with a gain of 1001, built according to the design rules proposed and tested for transient and frequency response, and CMRR, fulfilled the requirements in [7], including a CMRR of $123 \mathrm{~dB}$ at $50 \mathrm{~Hz}$.

\section{APPENDIX}

\section{Analysis of the Proposed AC COUPLing NeTwork}

Fig. 7 shows an alternative drawing of the proposed passive ac-coupling network in Fig. 2. Because it is a fully differential circuit, differential and common-mode voltages define four transfer functions. The main transfer function $G_{\mathrm{DD}}$ is the quotient between the Laplace transforms of the differential output voltage $V_{\mathrm{oD}}$ and the differential input voltage $V_{i D}$. Circuit analysis yields (6), as shown at the top of the page, where $\tau_{1}=R_{1} C$, $\tau_{1}^{\prime}=R_{1}^{\prime} C^{\prime}, \tau_{2}=R_{2} C$, and $\tau_{2}^{\prime}=R_{2}^{\prime} C^{\prime}$. If $\tau_{1}=\tau_{1}^{\prime}$, and $\tau_{2}=\tau_{2}^{\prime}, G_{\mathrm{DD}}$ reduces to

$$
G_{\mathrm{DD}}=\frac{s\left[s+\frac{1}{\tau_{1}+\tau_{2}}\right]}{\left[s+\frac{1}{\tau_{1}+\tau_{2}}\right]\left[s+\frac{1}{\tau_{2}}\right]}=\frac{1}{s+\frac{1}{\tau_{2}}}
$$

which is the same as (1). The zero-pole cancellation leading to (7) is not very sensitive to the matching condition. Considering unmatched passive components

$$
\begin{aligned}
R_{1}^{\prime} & =R_{1}+\Delta R_{1} \\
R_{2}^{\prime} & =R_{2}+\Delta R_{2} \\
C^{\prime} & =C+\Delta C
\end{aligned}
$$

we will have $\tau_{1} \neq \tau_{1}^{\prime}$ and $\tau_{2} \neq \tau_{2}^{\prime}$ and the transfer function (6) will display two zeros and two poles

$$
G_{\mathrm{DD}}(s)=\frac{s\left(s+z_{1}\right)}{\left(s+p_{1}\right)\left(s+p_{2}\right)}
$$

whose location depends on component values. A first-order approximation yields

$$
\begin{aligned}
& z_{1}=\frac{1}{\tau_{1}+\tau_{2}}\left[1-\frac{1}{2}\left(\frac{\Delta R_{1}+\Delta R_{2}}{R_{1}+R_{2}}+\frac{\Delta C}{C}\right)\right] \\
& p_{1}=\frac{1}{\tau_{1}+\tau_{2}}\left[1-\frac{1}{2}\left(\frac{\Delta R_{1}+\Delta R_{2}}{R_{1}+R_{2}}+\frac{\Delta C}{C}\right)\right] \\
& p_{2}=\frac{1}{\tau_{2}}\left[1-\frac{1}{2}\left(\frac{\Delta R_{2}}{R_{2}}+\frac{\Delta C}{C}\right)\right]
\end{aligned}
$$

which shows that variations in $R_{1}^{\prime}, R_{2}^{\prime}$, and $C^{\prime}$ with respect to $R_{1}, R_{2}$, and $C$, make $\mathrm{z}_{1}$ and $p_{1}$ to move in the same direction, so that there is still the pole-zero cancellation. Nevertheless, (10) is a first-order approximation and the cancellation depends on second-order effects. For example, if $R_{1}=R_{2}=5 \mathrm{M} \Omega$ and $C=1 \mu \mathrm{F}$, the singularities are

$$
z_{1}=-0.1000 \quad p_{1}=-0.1000 \quad p_{2}=-0.2000 .
$$

Assuming a $5 \%$ tolerance, $R_{1}^{\prime}=5.25 \mathrm{M} \Omega, R_{2}^{\prime}=5.25 \mathrm{M} \Omega$, $C^{\prime}=1.05 \mu \mathrm{F}$, and the zero and the poles move to

$$
z_{1}=-0.0955 \quad p_{1}=-0.0950 \quad p_{2}=-0.1909 .
$$

The variation in $\mathrm{z}_{1}, p_{1}$, and $p_{2}$ are around $5 \%$, as predicted by (10), whereas $\mathrm{z}_{1}$ is less than $0.5 \%$ apart from $p_{1}$.

\section{REFERENCES}

[1] R. Pallàs-Areny and J. G. Webster, "Common mode rejection ratio in differential amplifiers,” IEEE Trans. Instrum. Meas., vol. 40, pp. 669-676, Aug. 1991.

[2] A. C. Metting Van Rijn, A. Peper, and C. A Grimbergen, "Amplifiers for bioelectric events: a design with a minimal number of parts," Med. Bio. Eng. Comput., vol. 32, pp. 305-310, May 1994.

[3] R. Pallàs-Areny and J. G. Webster, Analog Signal Processing. New York: Wiley, 1999.

[4] J. C. Huhta and J. G. Webster, " $60-\mathrm{Hz}$ interference in electrocardiography," IEEE Trans. Biomed. Eng., vol. BME-20, pp. 91-101, Mar. 1973.

[5] M. J. Burke and D. T. Gleeson, “A micropower dry-electrode ECG preamplifier," IEEE Trans. Biomed. Eng., vol. 47, pp. 155-162, Feb. 2000 . 
[6] H. W. Smit, K. Verton, and C. A. Grimbergen, "A low-cost multichannel preamplifier for physiological signals," IEEE Trans. Biomed. Eng., vol. BME-34, pp. 307-310, Apr. 1987.

[7] American National Standard ANSI/AAMI EC38:1998, Ambulatory Electrocardiographs. Arlington, VA: Association for the Advancement of Medical Instrumentation, 1999.

[8] E. M. Spinelli, N. H. Martinez, and M. A. Mayosky, "A single-supply biopotential amplifier," Med. Eng. Phys., vol. 23, pp. 235-238, July 2001.

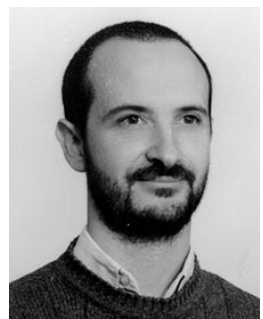

Enrique Mario Spinelli (S'02) was born in Balcarce, Argentina, in 1964. He received the Engineer and the M.S. degrees, both in electronics, from the Universidad Nacional de La Plata (UNLP), Argentina, in 1989 and 2000, respectively. He is currently working towards the Ph.D. degree at the Facultad de Ingeniería, Universidad Nacional de La Plata.

Since 1990, he has been with the Industrial Electronics, Control, and Instrumentation Laboratory (LEICI), Universidad Nacional de La Plata, working on scientific instrumentation. He is currently a Professor of control systems at the Facultad de Ingeniería, UNLP. His current interests are biomedical instrumentation and brain control interfaces.

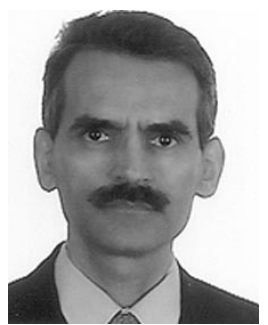

Ramon Pallàs-Areny ( $\left.\mathrm{M}^{\prime} 81-\mathrm{SM}^{\prime} 88-\mathrm{F}^{\prime} 98\right)$ received the Ingeniero Industrial and Doctor Ingeniero Industrial degrees from the Technical University of Catalonia (UPC), Barcelona, Spain, in 1975, and 1982, respectively.

$\mathrm{He}$ is currently a Professor of electronic engineering at the same university, where he teaches courses and does research in medical and electronic instrumentation. He is the author of several books on instrumentation in Spanish and Catalan, the latest one being Sensors and Interfaces, Solved Problems (Barcelona, Spain: Edicions UPC, 1999). He is also a coauthor (with J. G. Webster) of Sensors and Signal Conditioning, 2nd ed. (New York: Wiley, 2001) and Analog Signal Processing (New York: Wiley, 1999).

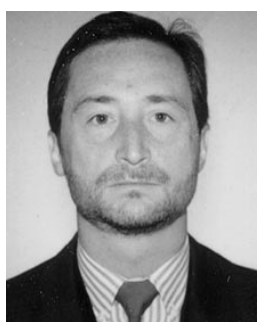

Miguel Angel Mayosky (M'97-SM'98) was born in La Plata, Argentina, in 1960. He received the Engineer on Electronics degree (First Class award) from the University of La Plata, La Plata, Argentina, and the Ph.D. degree in computer science from the $\mathrm{Au}-$ tonomous University of Barcelona, Spain, in 1983 and 1990, respectively.

He is currently a Full Professor of automatic control systems at the School of Engineering, the University of La Plata, and also a Member of the Research Staff of the Buenos Aires Scientific Research Commission (CICpBA), Buenos Aires, Argentina. His research activities involve real-time data acquisition and control systems, neural networks, and embedded computer architectures. 